\title{
Tornado Hazard and Exposure Model for Canadian Communities
}

\author{
Gabriel Narancio $^{1 *}$, Djordje Romanic ${ }^{1}$, Jubayer Chowdury ${ }^{1}$, Horia Hangan ${ }^{1}$ \\ ${ }^{1}$ WindEEE Research Institute, University of Western Ontario, London, Canada \\ *enaranci@uwo.ca
}

\begin{abstract}
Recently, on September 21st, 2018, an outbreak of six tornadoes with the strongest one being a high-end EF-3, devastated the Ottawa-Gatineau area in Canada causing damages that might approach US $\$ 100$ million. A set of experiments was performed in the WindEEE Dome tornado simulator at Western University to experimentally investigate the resilience of a Canadian community to tornado damages. A neighborhood in Dunrobin, Ontario that was ripped apart by an EF-3 tornado during the Ottawa-Gatineau tornado outbreak is chosen as a testbed for the study. The first goal is to experimentally replicate the conditions in the wind simulator that is as close as possible to the real event and provide an estimate of damages using the experimental data. The second goal is to perform a simple parametric study in the wind simulator and estimate the potential damage that would have been inflicted on the Dunrobin community by the same tornado (i.e., EF3) that traversed the community following a different path. Lastly, the potential influence of the different strengths of tornadoes on the Dunrobin community given the same footprint trajectory as the observed one will also be investigated. These experimentally determined damages will afterwards be compared against the losses reported by the insurance industries. This paper only focuses on the experimental technique behind this comprehensive study.
\end{abstract}

Tornado; extreme winds, sustainable \& resilient communities, experimental simulation, damage assessment, loss estimate, property insurance.

\section{INTRODUCTION}

Tornadoes are the most disastrous, violent and lethal wind events in the world. North America sees more tornadoes than any other place on the earth. In the United States (US) only, an average of 63 people dies every year and the damage in property averages US\$ 1123 billion [1]. Canada experiences on average 100 tornadoes every year.

Due to the increased variability of tornado events in recent years [2], it is expected that the insurance industries and homeowners will be more interested in tornado risk mitigation especially in regions that are particularly prone to tornadoes and extreme winds.
On September 21st, 2018, a tornado outbreak ripped through the Ottawa (Ontario) - Gatineau (Quebec) area. A total of six tornadoes, with the strongest one being a high-end EF-3 (wind speed up to $265 \mathrm{~km} / \mathrm{h}$ ), has been confirmed by Environment Canada (Figure 1). The outbreak left a path of destruction in its trail. 200 buildings were damaged of which more than 30 buildings were completely flattened in Dunrobin, Ontario [3,4]. 30 people were injured with no known fatalities. 150,000 homes and businesses were left without power and the power outage affected about 300,000 customers in and around the path of the tornadoes [5]. According to the Weekly Cat Report prepared by Aon Benfield, the total economic loss would approach US $\$ 100$ million.

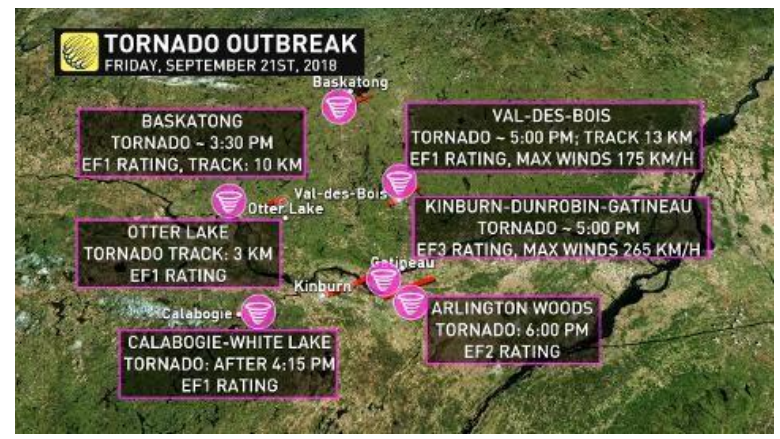

Figure 1: Location of the confirmed tornadoes (Source: Weather Network)

The EF-3 tornado that devastated Dunrobin, Ontario is the most powerful tornado seen in eastern Ontario since 1902. Damages were extensive with many buildings beyond repair (Figure 2). 


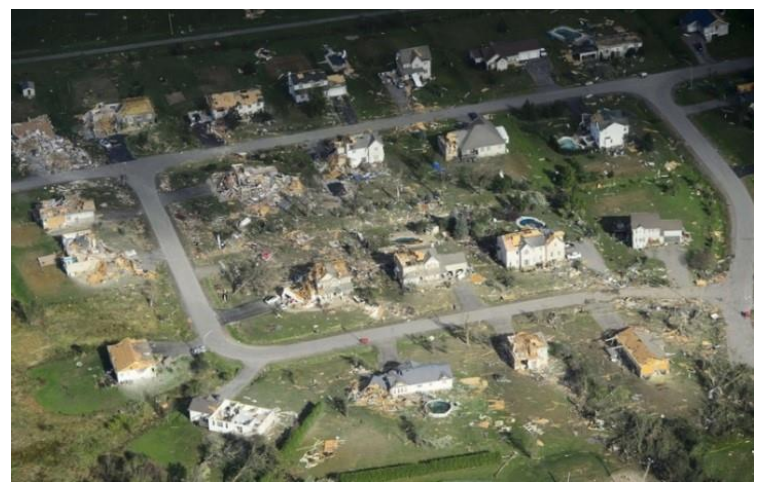

Figure 2: Damages from the EF-3 tornado in Dunrobin, Ontario (The Canadian Press/ Sean Kilpatrick)

This research proposes a set of experimental simulations of tornadoes affecting a neighborhood block shown in Figure 2. The experiments were carried out in the Wind Engineering, Energy and Environment (WindEEE) Dome simulator at Western University. The justifications for selecting this neighborhood in Dunrobin are mainly twofold: (1) the layout of the block is simple and can be treated as a typical residential layout in Canada; and (2) this community block has a small amount of tall trees and other obstacles that could serve as debris in tornadoes, thus making it simpler to directly relate the building damages with tornadic wind, rather than the damage being caused by flying debris. Wind loads on the selected buildings were measured for a model that was properly scaled to the scale of the tornado that passed through Dunrobin, Ontario.

Tornadic wind loads on isolated buildings in laboratory environments have been studied by several researchers. One of the earliest studies in this regard was performed by Jischke and Light [6] where tornadic wind loads on a rectangular model building were measured in a modified Ward's tornado simulator [7]. When compared with the atmospheric boundary layer (ABL) wind loads, significant changes in the forces and moments were reported for tornadic wind loads. Sengupta et al. [8] measured wind loads on a 1:100 scale model of a cubic building and a 1:500 scale model of a tall building in translating tornadoes in the Tornado/Microburst Laboratory Simulator at Iowa State University using force balance. The loads on the isolated buildings were found to be higher by a factor of 1.5 or greater in tornadoes (EF-2 or stronger) compared to the ASCE705 building standard [9]. Since the models were not instrumented with pressure taps, localized distributions of wind loads were missing. A 1:3500 scaled cubical model was tested in the Vortex Simulator II at Texas Tech University by Mishra et al. [10]. The F-4 rated tornado that hit Manchester, South Dakota on June 24, 2003, was replicated in the simulator at the model scale. The building model was equipped with 64 external surface pressure taps. Similar distributions of surface pressures as in ABL were observed for tornadic flow when the building was placed at a distance equal to the vortex core radius from the center. Haan et al. [11] also tested a 1:100 scale model of an isolated gable roof building with surface pressure taps in the tornado simulator at Iowa State University. Each pressure tap on the roof and the walls had a higher peak pressure coefficient compared to ASCE 7-05 [9]. In a recent study, Razavi and Sarkar [12] investigated the effect of different factors, such as translation speed and swirl ratio of a tornado, relative position, and orientation of the building concerning the direction of the tornado translation, on the wind loads on a low-rise building. A couple of the key observations was: (1) larger peak loads for lower swirl ratio; and (2) smaller peak loads with faster translation speeds. The literature review reveals that there is a clear gap in knowledge regarding tornadic wind loads on buildings at the community level, especially the effect of neighboring buildings on the tornadic wind loads on a building of interest.

Relating wind loads to damages is carried out through structural analysis. Ibrahim et al. [13] investigated the responses of pre-stressed concrete transmission line poles under tornado and downburst loads. Using an in-house developed finite element model the authors assessed damages and failure conditions of the transmission lines. They concluded that the pole can withstand an F2-rated tornado without collapsing. Savory et al. [14] carried out a dynamic structural analysis on a steel lattice tower to predict the failure conditions under tornado loads. They reported that the modes of failure in their study were similar to a limited number of field observations. Similar structural analysis based on experimentally obtained tornadic loads from the WindEEE Dome can be carried out to relate the wind-induced pressures to damage.

Losses caused by tornadoes - as well as any other natural peril — can be represented through a framework depicted in Figure 3. According to Romanic et al. [15], the factors that govern the loss are the climatology of tornadoes over the given area in terms of their frequency of occurrence, the strength of tornadoes and the geometry of their trajectories, the exposure map, and vulnerability that links the damage inflicted to a structure and wind speed. Vulnerability is an essential component in estimating losses because it directly links the natural hazard (i.e. tornado) and the economic loss inflicted on the building. This paper outlines the possibility of using a largescale tornado simulator-i.e., the WindEEE Dome-to quantitatively investigate the wind engineering aspects of the tornado loss framework portrayed in Figure 3.



Figure 3: Tornado loss assessment framework (modified after [15]).

Firstly, the building losses will be estimated for the neighborhood in Dunrobin, Ontario through the experiments performed at the WindEEE Dome and the tornado loss assessment framework shown in Figure 3. Secondly, the experimentally determined losses will be compared to the losses reported by the insurance companies. This experimental model could then be implemented for other communities in the tornado-prone regions to get an estimate of losses for different tornado scenarios. The results of the proposed study will also be the source material for future work that will focus on the construct of fragility and vulnerability curves for tornado damages using a tornado simulator. 


\section{METHODOLOGY}

\section{A. The WindEEE Dome}

The physical experiments carried out within the scope of this work were conducted at the Wind Engineering Energy and Environment (WindEEE) Dome [16,17] at Western University in London, Ontario. The WindEEE Dome is a three-dimensional wind testing facility designed to physically simulate nonstationary winds such as downbursts (micro- and macro-bursts), gusts, separated flows, and tornadoes. The facility is also fully capable of producing the stationary ABL winds at different scales $[16,17]$. The WindEEE Dome is a hexagonal chamber of $25 \mathrm{~m}$ in diameter surrounded by an outer return chamber of 40 $\mathrm{m}$ in diameter of the same hexagonal shape. A photograph of the WindEEE Dome testing chamber is shown in Figure 4. A total of 100 fans are positioned along the peripheral walls of the testing chamber, out of which 60 fans are installed in a matrix of 15 fans per row times 4 rows. This 60 -fan wall is used to produce different ABL winds. Additional systems, including an active boundary layer floor and moving bell-mouth allow for further manipulation of the flow. All these components are integrated via a sophisticated control system that allows manipulation of the flow with multiple degrees of freedom.

Tornado-like vortices in the WindEEE Dome are produced using 6 fans at the top chamber to provide updraft and periphery fans and/or vanes at a given intensity/angle at the lower chamber to generate rotation $[18,19]$. The tornado mode of the WindEEE Dome is schematically depicted in Figure 5. The bell-mouth connects the lower chamber to the upper one. The interplay between the suction caused by the upper fans and the swirl at the surface level produces tornado-like vortices of various sizes, intensities, and structures. A guillotine system suspended on two large beams (Figure 4) is used to translate the bell-mouth and consequently the tornadoes at a velocity of up to $2 \mathrm{~m} / \mathrm{s}$ over a $5 \mathrm{~m}$ distance. Flow visualization of a tornado is shown in Figure 6.

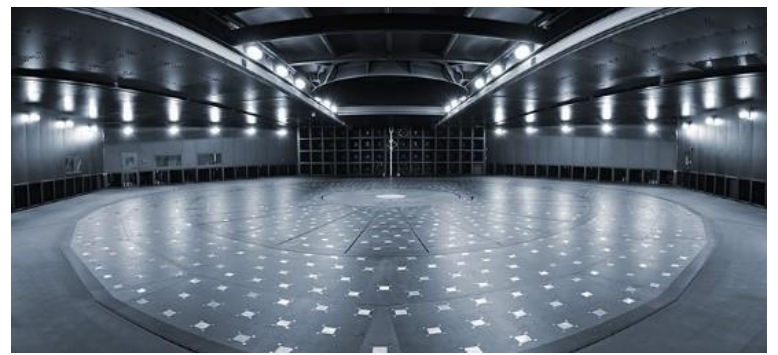

Figure 4: The WindEEE Dome testing chamber

In summary, the WindEEE Dome tornadic flow capabilities include:

- $\quad$ Replication of EF0-EF3 rated tornados.

- $\quad$ Properly scaled tornado flows [19,20].

- Geometric scale from approximately $1 / 50$ to about $1 / 200$

- $\quad$ Velocity scale of $1 / 3$ to $1 / 6$

- Variable swirl ratio
- Adjustable vortex diameter up to $4.5 \mathrm{~m}$

- $2 \mathrm{~m} / \mathrm{s}$ maximum tornado translation speed

- Floor roughness control

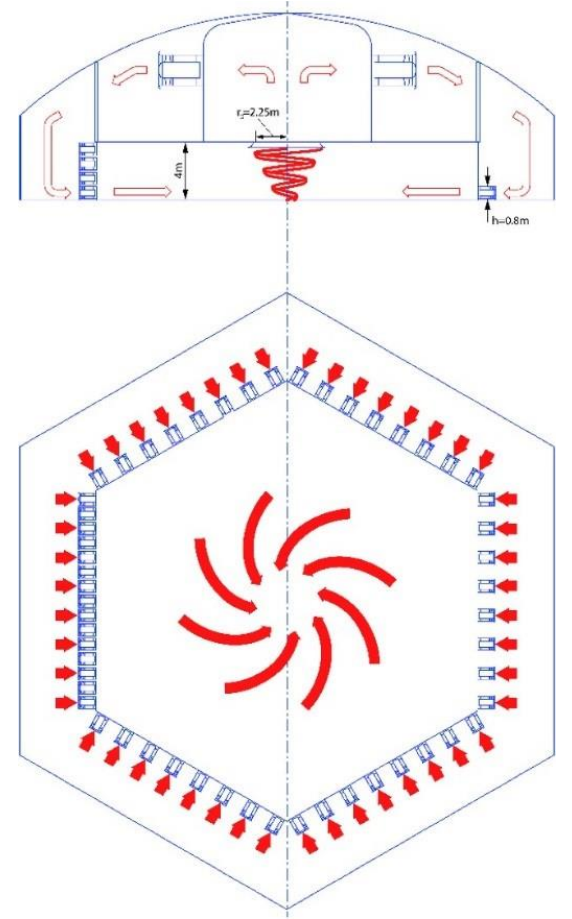

Figure 5: The WindEEE Dome tornado mode [19]



Figure 6: Flow visualization of a tornado in the WindEEE Dome.

\section{B. Measurement system}

The measurements of tornadic wind actions on the Dunrobin community were performed using a state-of-the-art pressure measurements system in the WindEEE Dome. The pressure scanners (developed by Pressure Systems Inc.) are differential pressure measurement units with an array of silicon piezoresistive pressure sensors, one for each pressure port. The maximum sampling rate of the system is $625 \mathrm{~Hz}$ per channel for a 32-channel pressure scanner. The surface pressures shall be expressed in terms of non-dimensional pressure coefficients $\left(C_{p}\right)$ as:

$$
C_{p, i}(t)=\frac{\left(p_{i}(t)-p_{r e f}\right)}{\frac{1}{2} \rho V_{r e f}^{2}}
$$

\footnotetext{
Supported by Mitacs and ICLR
} 
where $i$ is the $i$ th tap on the building model, $t$ is the time, $p_{i}$ is the measured pressure at the $i$ th tap (in inches of $\mathrm{H}_{2} \mathrm{O}$ ), $p_{\text {ref }}$ is the reference pressure, $\rho$ is the air density, and $V_{\text {ref }}$ is the reference velocity used to estimate the dynamic pressures. The proper choice of both $p_{\text {ref }}$ and $V_{\text {ref }}$ in experimentally produced tornadic flows is still an open question in the scientific community. This study shall use the undisturbed atmospheric pressure as $p_{\text {ref }}$ and the mean value of the maximum tangential velocity $V_{\text {tan.max }}$. Therefore, $V_{r e f}^{2}=V_{\text {tan,max }}^{2}$. This velocity is measured at $\left(r_{\text {tan,max }}, z_{\text {tan,max }}\right)$, where the former is the radius from the center of a tornado at which $V_{\text {tan.max }}$ is measured, and the latter is the height from the ground at which $V_{\text {tan.max }}$ is measured, respectively. These values are also used for the proper scaling of tornado-like vortices in the WindEEE Dome [20]. The velocity measurements will be carried out using a 3-hole Cobra Probe measuring probes that measure all three components of velocity.

Using the above approach, this study will document the time histories of $C_{p} s$ for each of the buildings in the Dunrobin community. These time histories will consequently be used to estimate the transient forces associated with the tornado passage over the community. The damage rates on each of the buildings will be estimated using the calculated forces from $C_{p} s$ and their tributary areas on the model.

\section{Test}

For the test, a scaled model of the Dunrobin community was constructed. The length scale of the tested model was 1:100. The eight buildings inside the block shown in Figure 2 were 3D printed with pressure taps on their surface. In addition to the pressure taps located in the building houses, pressure taps were also installed on the chamber floor to obtain ground pressures. In total, 1152 pressure taps were used. A picture of the model is shown in Figure 7.

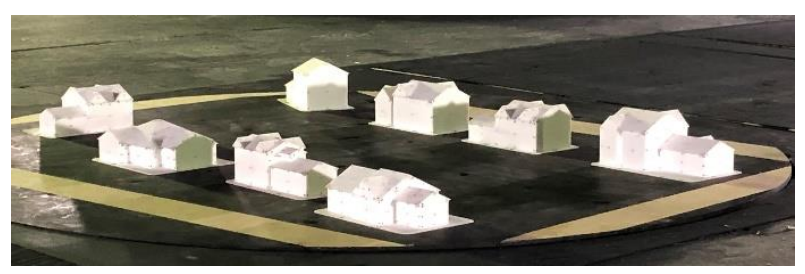

Figure 7: Image of the model

The buildings surrounding the analyzed block were built using polystyrene foam and included in the model as a proxy. The pressure measurements were recorded at a frequency of 500 $\mathrm{Hz}$ synchronized with the cobra probes. The pressure tubes that run from the surface of the buildings to the pressure scanners were all cut to the same length taking into consideration their frequency response.

Three different tornado categories were simulated: EF-1, EF2, and EF-3. Besides, three different tornado paths were considered. Firstly, we analyzed a case of a stationary tornado that is hovering over the block. Secondly, the actual path of the observed EF-3 tornado with the path direction $80^{\circ}$ from North, nearly WSW-ENE was simulated to replicate the observed conditions in the laboratory. Lastly, a path from SW-NE or $45^{\circ}$ from North was included as the typical path of tornadoes in these latitudes. These are depicted in Figure 8. The last direction is selected based on the knowledge that this is the most common path direction for tornadoes originated in North America [15]. For translating tornadoes, the measurement time was $20 \mathrm{~s}$ and for stationary $120 \mathrm{~s}$. The selected translation velocity for this test was $1.3 \mathrm{~m} / \mathrm{s}$. Every test was repeated 10 or 5 times to obtain more statistically significant data, according to the table below.


Figure 8: Tornado paths

The table below, shows all combinations tested.

\begin{tabular}{|c|c|c|l|c|}
\hline Tornado & $\begin{array}{c}\text { Offset } \\
(\mathbf{c m})\end{array}$ & Model Angle & Movement & Runs \\
\hline EF3 & -55 & 80 & Translation & 10 \\
\hline EF3 & -55 & 45 & Translation & 5 \\
\hline EF2 & -70 & 45 & Translation & 5 \\
\hline EF2 & -70 & 80 & Translation & 5 \\
\hline EF1 & -60 & 80 & Translation & 5 \\
\hline EF1 & -60 & 45 & Translation & 5 \\
\hline EF1 & -15 & 45 & Translation & 5 \\
\hline EF1 & -15 & 80 & Translation & 5 \\
\hline EF2 & -10 & 80 & Translation & 5 \\
\hline EF2 & -10 & 45 & Translation & 5 \\
\hline EF3 & +14 & 45 & Translation & 5 \\
\hline EF3 & +14 & 80 & Translation & 5 \\
\hline EF3 & 0 & 80 & Stationary & 1 \\
\hline EF2 & 0 & 80 & Stationary & 1 \\
\hline EF1 & 0 & 80 & Stationary & 1 \\
\hline EF3 & +83 & 80 & Translation & 5 \\
\hline EF3 & +83 & 45 & Translation & 5 \\
\hline
\end{tabular}

\footnotetext{
Supported by Mitacs and ICLR
} 


\section{ACKNOWLEDGMENT}

This research is supported by the Mitacs Accelerate funding and the Institute for Catastrophic Loss Reduction (ICLR).

\section{REFERENCES}

[1] Simmons, Kevin M., 2011. Economic and Societal Impacts of Tornadoes, University of Chicago Press Economics Books, University of Chicago Press, number 9781878220998, December. <https://ideas.repec.org/b/ucp/bkecon/9781878220998.html>

[2] Tippett, M.K., Lepore, C., Cohen, J.E., 2016. More tornadoes in the most extreme U.S. tornado outbreaks. Science 354, 1419-1423. https://doi.org/10.1126/science.aah7393

[3] Crawford, B., 2018. Tornado aftermath: "The roof fell on us" - Twisters leave residents awestruck and in the dark. Ott. Citiy.

[4] Gambrill, D., 2018. Two confirmed tornadoes blast Ottawa-Gatineau area. Can. Underwrit.

[5] Chianello, J., 2018. When is the power coming on? Not for days. CBC News.

[6] Jischke, M.C., Light, B.D., 1983. Laboratory simulation of tornadic wind loads on a rectangular model structure. J. Wind Eng. Ind. Aerodyn. 13, 371-382. https://doi.org/10.1016/0167-6105(83)90157-5

[7] Church, C.R., Snow, J.T., Agee, E.M., 1977. Tornado Vortex Simulation at Purdue University. Bull. Am. Meteorol. Soc. 58, 900-909. https://doi.org/10.1175/1520-0477(1977)058<0900:TVSAPU>2.0.CO;2

[8] Sengupta, A., Haan, F.L., Sarkar, P.P., Balaramudu, V., 2008. Transient loads on buildings in microburst and tornado winds. J. Wind Eng. Ind. Aerodyn. 96, 2173-2187. https://doi.org/10.1016/j.jweia.2008.02.050

[9] ASCE 7-05, 2006. Minimum Design Loads for Buildings and Other Structures, Standards. American Society of Civil Engineers.

[10] Mishra, A.R., James, D.L., Letchford, C.W., 2008. Physical simulation of a single-celled tornado-like vortex, Part B: Wind loading on a cubical model. J. Wind Eng. Ind. Aerodyn. 96, 1258-1273. https://doi.org/10.1016/j.jweia.2008.02.027

[11] Haan, F.L., Balaramudu, V.K., Sarkar, P.P., 2010. Tornado-Induced Wind Loads on a Low-Rise Building. J. Struct. Eng. 136, 106-116. https://doi.org/10.1061/(ASCE)ST.1943-541X.0000093

[12] Razavi, A., Sarkar, P.P., 2018. Tornado-induced wind loads on a low-rise building: Influence of swirl ratio, translation speed and building $\begin{array}{lllll}\text { parameters. } & \text { Eng. } & \text { Struct. } & 167, & 1-12 .\end{array}$ https://doi.org/10.1016/j.engstruct.2018.03.020

[13] Ibrahim, A.M., El Damatty, A.A., El Ansary, A.M., 2017. Finite element modelling of pre-stressed concrete poles under downbursts and tornadoes. Eng. Struct. 153, 370-382. https://doi.org/10.1016/j.engstruct.2017.10.047

[14] Savory, E., Parke, G.A.R., Zeinoddini, M., Toy, N., Disney, P., 2001. Modelling of tornado and microburst-induced wind loading and failure of a lattice transmission tower. Eng. Struct. 23, 365-375. https://doi.org/10.1016/S0141-0296(00)00045-6

[15] Romanic, D., Refan, M., Wu, C.-H., Michel, G., 2016. Oklahoma tornado risk and variability: A statistical model. Int. J. Disaster Risk Reduct. 16, 19-32. https://doi.org/10.1016/j.ijdrr.2016.01.011

[16] Hangan, H., Refan, M., Jubayer, C., Parvu, D., Kilpatrick, R., 2017a. Big Data from Big Experiments. The WindEEE Dome, in: Pollard, A., Castillo, L., Danaila, L., Glauser, M. (Eds.), Whither Turbulence and Big Data in the 21st Century? Springer International Publishing, pp. 215-230. https://doi.org/10.1007/978-3-319-41217-7_12

[17] Hangan, H., Refan, M., Jubayer, C., Romanic, D., Parvu, D., LoTufo, J., Costache, A., 2017b. Novel techniques in wind engineering. J. Wind Eng. Ind. Aerodyn. 171, 12-33. https://doi.org/10.1016/j.jweia.2017.09.010

[18] Ashrafi, A., Romanic, D., Chowdhury, J., Hangan, H., 2018. Producing $1 / 100$ and larger scale tornadoes in a wind simulator. Presented at the Tornado Hazard Wind Assessment and Reduction Symposium (THWARTS 2018), Champaign, IL.

[19] Refan, M., Hangan, H., 2018. Near surface experimental exploration of tornado vortices. J. Wind Eng. Ind. Aerodyn. 175, 120-135. https://doi.org/10.1016/j.jweia.2018.01.042

[20] Refan, M., Hangan, H., Wurman, J., 2014. Reproducing tornadoes in laboratory using proper scaling. J. Wind Eng. Ind. Aerodyn. 135, 136148. https://doi.org/10.1016/j.jweia.2014.10.008 